Rev. Fac. Med. vet. Zootec. Univ. S. Paulo $12: 27-32,1975$

\title{
DISTÂNCIA DO TORUS PYLORICUS A PAPILLA DUODENI MAJOR E DESTA A PAPILLA DUODENI MINOR EM BOVINOS DE ORIGEM EUROPEIA E INDIANA §
}

\author{
Orlando M. PAIVA * \\ Vicente BORELLI ** \\ José PEDUTI NETO ***
}

\section{RFMV-A/3}

\begin{abstract}
Paiva, O. M.; Borelli, V.; Peduti Neto, J. - Distância do torus pyloricus $\grave{a}$ papilla duodeni major e desta à papilla duodeni minor em bovinos de origem européia e indiana. Rev. Fac. Med. vet. Zootec. Univ. S. Paulo, 12:27-32, 1975.

Resumo: A distância entre o torus pyloricus $e$ a papilla duodeni major, bem como a distância entre esta e a papilla duodeni minor, foram medidas em 117 tratos intestinais de bovinos, adultos; 60 Bos indicus e 57 Bos taurus. $A$ análise estatistica dos valores obtidos não mostrou diferencas significantes atinentes aos sexos, mas, evidenciou-as, ao compararmos 08 dados encontrados para Bos indicus e Bos taurus.

O espaço compreendido do torus pyloricus à papilla duodeni minor varia independentemente.

Estes resultados corroboram a idéia de que os bovinos de origem indiana $e$ européia apresentam diferentes comprimentos de intestino.

Unitermos: Anatomia*; Intestinos*; Papilas duodenais*; Bovinos*; A parelho digestivo.
\end{abstract}

\section{INTRODUÇÃO E LITERATURA}

Investigacões relativas às distâncias que separam o torus pyloricus da papilla duodeni hepática (PAIVA \& ASSIS RIBEI$\mathrm{RO}^{22}$ - 1948) e esta da papilla duodeni pancreatica (PAIVA \& D'ERRICO ${ }^{25}$ 1949), levadas a cabo há alguns anos, por um de nós, em bovinos azebuados, nāo permitiram fossem apuradas, estatisticamen- te, as eventuais diferenças ligadas ao sexo e menos ainda as decorrentes de confronto com dados estabelecidos para animais de origem européia. A tais proposiçōes, agora objeto de nosso interesse, acrescentamos novo item, ou seja, o estudo de correlação dessas medidas, em taurinos e zebuinos.

8. Comunicado ao V Congresso Brasileiro de Anatomia, realizado em São Paulo, de 10 a 13 de julho de 1967.

* Catedrático.

* Professor Adjunto.

** Professor Assistente Doutor. Departamento de Cirurgia e Obstetricia da Faculdade de Medicina Veterinária e Zootecnia da USP. 
PAIVA, O. M.; BORELLI, V.; PEDUTI NETO, J. - Distância do torus pyloricus à papilla duodeni major e desta à papilla duodeni minor em bovinos de origem européia e indiana. Rev. Fac. Med. vet. Zootec. Univ. S. Paulo, 12:27-32, 1975.

PAIVA \& ASSIS RIBEIRO22 realizaram a mensuração de 411 segmentos duodenais, retirados de 312 machos e 14 fêmeas, cujas idades variavam de 28 a 51 meses, aproximadamente; os 85 restantes provieram de espécimes não identificados. Das pecas utilizadas, os AA. selecionaram, ao acaso, 144 pertencentes a machos e mais 20 , do referido grupo de 85 , visando medi-las a fresco e após fixação pelo formol; no material não submetido a tratamento, a distância da extremidade intestinal do toro pilórico à abertura do ducto colédoco atingiu $45,02 \mathrm{~cm} \pm 0,49$.

PAIVA \& D'ERRICO ${ }^{25}$ valeram-se de 324 tratos duodenais, isolados de 231 machos, 15 fêmeas e de 78 exemplares sobre os quais faltavam informações precisas. Do primeiro e segundo lotes, integrados por indivíduos com idades que se distribuíram entre 28 e 51 meses, mais ou menos, apartaram 109 peças, isto é, respectivamente, 103 e 6, destinando-as à tomada de medidas, anterior e ulteriormente à ação do formol; a distância da papila duodenal maior à papila duodenal menor, apontada nas peças frescas, foi de $27,8 \mathrm{~cm} \pm 0,51$.

As diferenças patenteadas, em face do que divulgam livros de texto e publicacões especiais, alvitram PAIVA \& ASSIS RIBEIRO $^{22}$ e PAIVA \& D'ERRICO ${ }^{25}$, nestes dois trabalhos, dependeriam, possivelmente, do menor comprimento do intestino dos zebuinos, fato depois confirmado por PAIVA, BORELLI \& PEDUTI NETO ${ }^{24}$ (1967), ao servirem-se, inclusivamente, de anterior pesquisa de PAIVA \& BORELLI ${ }^{23}$ (1962).

Noticias referentes a bovinos de origem, provavelmente européia, encontramo-las em BAUMANN \& SCHMOTZER ${ }^{2}$ (1912), MANN, BRIMHALL \& FOSTER ${ }^{17}(\mathbf{1 9 2 0})$ MANN, FOSTER \& BRIMHALL ${ }^{18}$ (1920), FAVILLI ${ }^{10}$ (1923), DELPRATO ${ }^{8}$ (1938), THOMSON $^{28}(1940)$.

BAUMANN \& SCHMOTZER ${ }^{2}$, com base em 8 observações, dizem que a desembocadura do colédoco no duodeno faz-se a $60.7 \mathrm{~cm}$ do piloro; a do ducto pancreático localiza-se, distalmente, a $32,6 \mathrm{~cm}$ dela.

MANN, BRIMHALL \& FOSTER ${ }^{17}$ procuram esclarecer dois fatos: a) se o trato biliar extra-hepático compensa a falta de vesícula biliar; b) se existe relação entre a presença e ausência deste reservatório e a distância que separa o piloto do ponto de chegada, no duodeno, do ducto biliar comum. Para tanto, registraram a distância do piloro à entrada do ducto no intestino, em bovinos, ovinos, caprinos, caninos, felinos, leporinos e eqüinos, cada uma das espécies representada por 5 exemplares, praticamente do mesmo tamanho; nas vacas examinadas, os AA. obtiveram as seguintes medidas: $500,550,575$ e 550 mm. Ainda a respeito de bovinos, afirmam que o ducto biliar comum penetra a $50-70 \mathrm{~cm}$ do piloro.

MANN, FOSTER \& BRIMHALL ${ }^{18}$ focalizando a relação entre o ducto biliar comum e o ducto pancreático, em animais domésticos e de laboratório, ilustram de forma esquemática a disposiçāo dessas vias excretoras, nos bovinos, indicando-as como a se abrirem no duodeno, respectivamente, a 575 e a $875 \mathrm{~mm}$ do piloro.

FAVILLI ${ }^{10}$, ocupando-se do colédoco, comenta que é visto a alcançar a parede intestinal, independentemente, a discreta distância do piloro, vale dizer, entre 3 $\left(\right.$ LAVOCAT $\left.^{14}\right)$ e 6 ou $7 \mathrm{~cm}\left(\right.$ COLIN $\left.^{7}\right)$; quanto ao ducto excretor pancreático, acrescenta, acha-se cerca de 30 ou $40 \mathrm{~cm}$ a jusante do colédoco.

DELPRATO ${ }^{8}$, descrevendo as vias biliares extra-hepáticas e suas variacões, esclarece que o colédoco lança-se no duodeno a uns 60 a $70 \mathrm{~cm}$ do piloro.

THOMSON ${ }^{28}$, cuidando das vias biliares extra-hepáticas, em algumas ordens de mamíferos, explica que, nos bovinos, ao ducto biliar comum corresponde papila situada $61 \mathrm{~cm}$ caudalmente ao piloro.

No tocante à espécie bovina, os tratadistas fornecem-nos números discordantes para a distância do torus pyloricus à papilla duodeni major. Assim, FAVILLI ${ }^{11}$ (1931) avaliou-a em 30,0 a $70,0 \mathrm{~cm}$; ELLENBERGER \& BAUM ${ }^{9}$ (1932), MARTIN \& SCHAUDER ${ }^{19}$ (1935), MÁSSUI ${ }^{20}$ (1960), AKAJEWSKI ${ }^{1} \quad(1962)$ calcularam-na em 50,0 a $70,0 \mathrm{~cm}$; BRUNI \& ZIMMERL $^{3}$ (1947), em 60,0 a $75,0 \mathrm{~cm}$; GONZALEZ Y GARCIA \& ALVAREZ12 (1929), em 62,0 a $70,0 \mathrm{~cm}$; LESBRE ${ }^{16}$ (1922), CARADONNA ${ }^{4}$ (in ZIMMERL, 1929), ambos citando COLIN? em 62,0 a $75,0 \mathrm{~cm}$; MONTANE \& BOURDELLE 21 (1917) em 65,0 a 70,0 cm; SISSON ${ }^{16}$ (1933), SISSON \& GROSSMAN ${ }^{27}$ (1965), em $60,0 \mathrm{~cm}$; LEPOUTRE ${ }^{15}(1921)$ em 60,0 cm e mais. 
PAIVA, O. M.; BORELLI, V.; PEDUTI NETO, J. - Distância do torus pyloricus à papilla duodeni major e desta à papilla duodeni minor em bovinos de origem européia e indiana. Rev. Fac. Med. vet. Zootec. Univ. S. Paulo, 12:27-32, 1975.

Os dados concernentes à distância entre a papilla duodeni major e a papilla duodeni minor, todavia, são algo menos desiguais. De fato, FAVILLI ${ }^{11}$, ELLENBERGER \& BAUM ${ }^{9}$ MARTIN \& SCHAUDER $^{19}$, MASSUI 20, AKAJEWSKI, KA$\mathrm{TO}^{13}$ (1963), estimaram-na em 30,0 a 40,0 cm: CARADONNA \& BOSSI ${ }^{5}$ (in BOSSI, CARADONNA SPAMPANI, VARALDI \& ZIMMERL - s.d.), LEPOUTRE ${ }^{15}$, LESBRE ${ }^{16}$ CARADONNA ${ }^{4}$ (in ZIMMERL), GONZÁLEZ Y GARCIA \& ALVAREZ ${ }^{12}$, BRUNI \& ZIMMERL ${ }^{3}$, em 35,0 a $40 \mathrm{~cm}$; MONTANE \& BOURDELLE 21 , em 35,0 a 40,0 ou $60,0 \mathrm{~cm}$; $\operatorname{SISSON}^{26}$ e SISSON \& GROSSMAN 27 , em $30,0 \mathrm{~cm}$.

\section{MATERIAL E METODO}

O material de que nos valemos compõe-se de 117 tractos duodenais, pertencentes a 57 taurinos, 21 machos e 36 fêmeas, abatidos no Matadouro Municipal de Alegrete (Rio Grande do Sul) e a 60 zebuínos, 30 machos e 30 fêmeas, sacrificados no Frigorífico de Cotia (São Paulo), todos com dentição definitiva, por outras palavras, com idades superiores a 60 (bovinos de origem européia) e 52 meses (bovinos de origem indiana - CHIEFI, PAIVA \& VEIGA ${ }^{6}, 1948$ ), aproximadamente.

Para isolar a peça, eram praticados dois cortes transversais, completos: o primeiro em plena regiāo pilórica do abomaso e o segundo cerca de 120 centímetros distalmente ao piloro; a seguir, seccionavam-se o pequeno omento e mesoduodeno, junto à víscera. Uma vez desfeitas, cuidadosamente, as pregas da serosa, abria-se o duodeno ao longo do contorno livre, no próprio local da colheita, removendo-se, entāo, os resíduos alimentares da mucosa, por meio de jato de água sob pequena pressão. Dos segmentos assim preparados, dispostos sobre superfície plana, foram tiradas medidas, a fresco, por um de nós (BORELLI), com auxílio de régua, à qual adaptamos duas hastes paralelas, uma fixa e outra móvel, consoante estabelecido por PAIVA \& ASSIS RIBEIRO ${ }^{22}$ e PAIVA \& D'ERRICO ${ }^{25}$

\section{R E S U L T A D O S}

Seguido o critério exposto, encontramos para a distância entre o torus pyloricus e a papilla duodeni major, em machos e fêmeas, respectivamente, as seguintes médias e desvios padrões: taurinos $-45,74 \mathrm{~cm} \pm$ 5,65 e $47,47 \mathrm{~cm} \pm 6,18$; zebuínos $-43,52$ $\mathrm{cm} \pm 6,05$ e $41,59 \mathrm{~cm} \pm 5,97$. Os dados relativos à distância entre a papilla duodeni major e a papilla duodeni minor, nos mesmos grupos de animais, mantida a precedente seqüência, foram: $30,86 \mathrm{~cm} \pm$ 5,69 e $33,83 \mathrm{~cm} \pm 6,22 ; 29,40 \mathrm{~cm} \pm 4,74$ e $28,92 \mathrm{~cm} \pm 5,73$. As diferenças correspondentes a machos e fêmeas, analisadas pelo "teste t", revelaram-se, por ordem de citação, iguais a 1,03 e 1,23 , no tocante à distância do piloro à abertura do colédoco e a 1,77 e 0,35 , relativamente à interpapilar, valores não significantes, ao nível de rejeição adotado (5\%). Se, entretanto, considerarmos conjuntamente machos e fêmeas, as médias globais registradas, isto é, $46,84 \mathrm{~cm} \pm 6,38$ e $42,56 \mathrm{~cm} \pm 6,06$, para a distância do torus pyloricus à papilla duodeni major e $32,73 \mathrm{~cm} \pm 6,20$ e 29,16 $\mathrm{cm} \pm 5,26$, para a compreendida entre esta e a papilla duodeni minor, em taurinos e zebuínos, respectivamente, evidenciam, quando submetidas a comparação pelo "teste t", valores (3,69 no primeiro caso e 3,34 no segundo) que traduzem a existência de diferenças significantes ao nível de rejeição adotado $(5 \%)$.

Enfim, estudo estatístico de confronto das duas medidas assinaladas, em cada animal, permite-nos verificar que nāo se correlacionam, quer nos machos quer nas fêmeas, examinados separadamente bovinos de origem européia e indiana.

\section{COM E N T ÁRIOS}

As informaçōes dos tratadistas MONTANE \& BOURDELLE ${ }^{21}$, LEPOUTRE ${ }^{15}$ e LESBRE $^{16}$; as de CARADONNA ${ }^{4}$ (in ZIMMERL); as de FAVILLI ${ }^{11}$ e GONZALEZ Y GARCIA \& ALVAREZ ${ }^{12}$; as de ELLENBERGER \& BAUM ${ }^{9}$ e SISSON $^{26}$; as de MARTIN \& SCHAUDER ${ }^{19}$, BRUNI \& ZIMMERL ${ }^{3}$, MASSUI ${ }^{20}$ AKAJEWSKI KATO ${ }^{13}$; mais as de SISSON \& GROSSMAN ${ }^{27}$, CARADONNA \& BOSSI $^{5}$ (in BOSSI, CARADONNA, SPAMPANI, VARALDI \& ZIMMERL) e, ainda, as de LAVOCAT $^{14}$ e COLIN $^{7}$ (cit. FAVILLI 1923), todas referentes às distâncias torus pyloricus $\mathrm{x}$ papilla duodeni major $(30,0-$ $75,0 \mathrm{~cm}$ ) e papilla duodeni major $\mathrm{x}$ papilla duodeni minor $(30,0-60,0 \mathrm{~cm})$, obtidas em soma ignorada de espécimes, não po- 
PAIVA, O. M.; BORELLI, V.; PEDUTI NETO, J. - Distância do torus pyloricus à papilla duodeni major e desta à papilla duodeni minor em bovinos de origem européia e indiana. Rev. Fac. Med. vet. Zootec. Univ. S. Paulo, 12:27-32, 1975.

dem, por isso mesmo, prestar-se a qualquer tipo de cotejo. Cabe, aliás, o mesmo reparo, no que tange aos números veículados pelos trabalhos de MANN, FOSTER \& BRIMHALL ${ }^{18}$ (papilla duodeni major $57,5 \mathrm{~cm}$ e papilla duodeni minor - 87,5 cm), FAVILLI ${ }^{11}$ (papilla duodeni minor 30,0 ou $40,0 \mathrm{~cm}$ ), DELPRATO ${ }^{8}$ ( papilla duodeni major $-60,0$ a 70,0 cm) e THOMSON 8 (papilla duodeni major - 61,0 $\mathrm{cm})$. Por outro lado, a falta de dados sobre o sexo e a idade dos animais impede-nos, também, de discutir os resultados de BAUMANN \& SCHMOTZER ${ }^{2}$ (papilla duodeni major - 60,7 cm e papilla duodeni minor - $32,6 \mathrm{~cm}$ ) e os de MANN, BRIMHALL \& FOSTER ${ }^{17}$ (papilla duodeni major $-50,7$ a $57,5 \mathrm{~cm}$ e 50,0 a 70,0 cm).

Contrapondo-se, todavia, mediante o "teste t", a média indicada por PAIVA \& ASSIS RIBEIRO $22(45,02 \mathrm{~cm})$ para a distância entre toro pilórico e papila duodenal maior, em mestiços de zebu, com as médias que apuramos, relativamente a taurinos $(46,84 \mathrm{~cm})$ e zebuinos $(42,56 \mathrm{~cm})$, achamos os valores 1,93 e 2,65; evidenciamos, assim, diferença estatisticamente significante ao nível de $5 \%$, apenas no concernente a bovinos de origem indiana.

Comparando-se, afinal, por meio do "teste t", a média estabelecida por PAIVA \& D'ERRICO ${ }^{25}(27,80 \mathrm{~cm})$ para a distância interpapilar, em azebuados, com as correspondentes médias que determinamos nas peças de taurinos $(32,73 \mathrm{~cm})$ e zebuinos $(29,16 \mathrm{~cm})$, verificamos, ante os valores calculados, ou seja, 5,09 e 1,60, a ocorrência de diferença estatisticamente significante, ao nível de $5 \%$ somente no primeiro dos confrontos.
As contradiçōes ora patenteadas, considerando-as que PAIVA \& ASSIS RIBEIRO $^{22}$ e PAIVA \& D'ERRICO ${ }^{25}$ se utilizaram de peças provenientes na sua maioria de machos, cremos devam correr à conta da amostragem; afora isso, consoante demonstramos, as duas mensuraçōes levadas a efeito não se correlacionam.

Assim, havendo comprovado que as distâncias objeto deste estudo variam independentemente, escapam à influência do sexo e são, nos bovinos de origem indiana, mais curtas, entendemos procedente a suposição dos citados AA., segundo a qual a última das observaçōes estaria ligada ao menor comprimento do intestino dos azebuados, questão dirimida em definitivo, graças às investigaçōes de PAIVA \& BORELLI $^{23}$ e PAIVA, BORELLI \& PEDUTI NETO ${ }^{24}$.

\section{O N C L U S O E S}

Os resultados expostos permitem-nos as seguintes conclusões:

1) a distância entre o torus pyloricus e a papilla duodeni major, bem como a distância entre esta e a papilla duodeni minor, não diferem, significantemente, em machos e fêmeas, quer no Bos taurus, quer no Bos indicus;

2) as distâncias torus pyloricus $\mathbf{x} p a$ pilla duodeni major e papilla duodeni major $\mathbf{x}$ papilla duodeni minor diferem significantemente, quando confrontamos bovinos de origem européia e indiana;

3) as duas medidas tomadas em cada peça variam independentemente, seja nos machos, seja nas fêmeas, ao considerarmos, em separado, taurinos e zebuínos. 
PAIVA, O. M.; BORELLI, V.; PEDUTI NETO, J. - Distância do torus puloricus à papilla duodeni major e desta à papilla duodeni minor em bovinos de origem européia e indiana. Rev. Fac. Med. vet. Zootec. Univ. S. Paulo, 12:27-32, 1975.

RFMV-A/3

Paiva, O. M.; Borelli, V.; Peduti Neto, J. - Distances from the torus pyloricus to the papilla duodeni major and from that to the papilla duodeni minbr, in Bos taurus and Bos indicus. Rev. Fac. Med. vet. Zootec. Univ. S. Paulo, 12:27-32, 1975

SUmmary: The distance between the torus pyloricus and the papilla duodeni major as well as the distance between the latter and the papilla duodeni minor were obtained from 117 intestines of adult cattle: 60 were Bos indicus and 57 Bos taurus.

The statistical analysis of these data have shown no significant diffe rences between sexes but a marked one between the species (Box indicus $x$ Bos taurus).

The distances between torus pyloricus and papilla duodeni major and papilla duodeni minor vary independently.

These results corroborate the idea that the lenght of the intestines of cattle from European and Indian origin are different.

UNITERMS: Anatomy*; Digestive system; Intestine*; Duodenal papilla*; Bovine.

\section{REFERENCIAS BIBLIOGRAFICAS}

1. AKAJEWSKI, A. I. - Anatomia dos animais domésticos - Moscou, Editora de Literatura Agrícola, 1962.

2. BAUMANN, A. \& SCHOMOTZER, B. Beitrage zur vergleichenden Anatomie des Vater'schen Divertikels und der Gallen - und Penkressgonge. Ost. Wschr. Tierheilk., $37(47): 469-71$; (48) :479-81; (51):523-24, 1912

3. BRUNI, A. C. \& ZIMMERL, U. - Anatomia degli animali domestici. Milano, Francesco Vallardi, 1947, v. 2.

4. CARADONNA, G. B. - Apparecchio Gastropolmonare. In: ZIMMERL, U Trattato di anatomia veterinaria. Milano, Francesco Vallardi, 1929, v. 2.

5. CARADONNA, G. B. \& BOSSI, V. Splanenologia. In: BOSSI, V.; CARADONNA, G. B.; SPAMPANI, G.; VARALUI, L.; ZIMMERL, U, Trattato di anatomia veterinaria. Milano, Francesco Vallardi, 1909, v. 2, p. 435938.

6. CHIEFFI, A.; PAIVA, O. M.; VEIGA J. S. - Contribuição para o estudo da cronologia dentária no Zebu. Rev. Fac. Med. vet., S. Paulo, 3(4):251-69, 1948 .
7. COLIN - Apud CARADONNA, G. B. 1929; FAVILLI, N., 1923; LESBRE, F. X., 1922 .

8. DELPRATO, I. O. - Las vias biliare extrahepaticas del bovino y sus variaciones. An. Fac. Med. vet. La Plata, 1(1):37-47, 1938.

9. ELLENBERGER, W. \& BAUM, H. Handbuch der vergleichenden Anato mie der Haustiere. 17 Auf. Berlin, Julius Springer, 1932.

10. FAVILLI, N. - L'Anatomia del fegato nei principali animali domestici (cavallo, bue, maiale, cane) - Pisa, Cesari, 1923.

11. FAVILLI, N. - Nozioni comparate di anatomia e fisiologia degli animal rurali. Torino, Unione Tipografico Editrice Torinese, 1931.

12. GONZALEZ Y GARCIA, J. \& ALVAREZ R. G. - Anatomia comparada de los animales domesticos. 3. ed. Zaragoze, La Academica, 1929.

13. KATO, K. - Katiku hikaku kaibon zusitвu. Exposição gráfica da anatomia dos animais domésticos. 6. ed. Tokio Yokando, 1963, v. 2 
PAIVA, O. M.; BORELLI, V.; PEDUTI NETO, J. - Distância do torus pyloricus à papilla duodeni major e desta a papilla duodeni minor em bovinos de origem européia e indiana. Rev. Fac. Med. vet. Zootec. Univ. S. Paulo, 12:27-32, 1975.

14. LAVOCAT. - In: FAVILLI, N., 192310.

15. LEPOUTRE, L. - Notes du cours d'anatomie comparée des animaux domestiques. Gembloux, J. Duculot, 1921.

16. LESBRE, F. X. - Précis d'anatomie comparée des animaux domestiques. Paris, J. B. Baillière, 1922, v. 1.

17. MANN, F. C.; BRIMHALL, S. D.; FOS TER, J. P. - The extrahepatic biliary tract in common domestic and laboratory animals. Anat. Rec., 18(1):47-66 1920.

18. MANN, F. C.; FOSTER, J. P.; BRIMHALL, S. D. - The relation of the common bile duct to the pancreatic duct in common domestic and laboratory animals. J. Lab. clin. Med., 5 (4) :203-6, 1920.

19. MARTIN, P. W. \& SCHAUDER, W. Lehrbuch der Anatomie der Haustiere. 3 Auf. Stuttgart, Schickhart \& Ebner, 1935 , v. 3 , t. 2 .

20. MASSUI, K, - Katiku hikaku Kaibon gaku. Anatomia comparada dos animais domésticos. 7. ed. Tokio, Yokendo, 1960 , v. 2 .

21. MONTANE, L. \& BOURDELLE, E. Anatomie régionale des animaux domestiques. Paris, J. B. Baillière, 1917, v. 2 .

22. PAIVA, O. M. \& ASSIS RIBEIRO, P. Distância do torus pyloricus d papilla duodeni hepatica em bovinos mestiços de zebu. Rev. Fac. Med. vet., S. Paulo, $3(4)$ : $223-46,1948$

23. PAIVA, O. M. \& BORELLI, V. - Comprimento total do intestino em bovinos azebuados. [Trabalho comunicado à Conferência Anual da Sociedade Paulista de Medicina Veterinária, 17, 1962 J.

24. PAIVA, O. M.; BORELLI, V.; PEDUT NETO, J. - Comprimento total do intestino em bovinos de origem européia. [Trabalho comunicado à Conferêneia Anual da Sociedade Paulista de Medicina Veterinária, 20, 1965].

25. PAIVA, O. M. \& D'ERRICO, A. A. Distância da papilla duodeni hepática à papilla duodeni pancreática em bovinos mestiços de zebu. Rev. Fac. Med. vet., S. Paulo, 4(1):5-26, 1949.

26. SISSON, S. - Anatomia de los animales domesticos. Barcelona, Salvat, 1933

27. SISSON, S. \& GROSSMAN, J. D. Anatomia de los animales domesticos. 4. ed. Barcelona, Salvat, 1965.

28. THOMSON, S. C. - Studies of the anatomy of the extrahepatic biliary tract in mammalia. Zool. Ser. Field Mus. Nat. Hist., $22(6): 415-30,1940$.

Recebido para publicação em 27-7-75

Aprovado para publicação em 17-9-75 